\title{
Pseudoautosomal Region
}

National Cancer Institute

\section{Source}

National Cancer Institute. Pseudoautosomal Region. NCI Thesaurus. Code C42296.

The human Y chromosome is composed of two different parts: a pseudoautosomal region that is homologous to a region of the $\mathrm{X}$ chromosome and which is responsible for sex chromosome pairing and a Y-specific part that encodes the sex determining gene. Genes within the pseudoautosomal region are not sex linked. 